\title{
Influence of Polarization Mode Dispersion on Optical Communication Network at High Bit Rate 10 Gbps
}

\author{
R. S. Habeb \\ Department of Physics / College of Education, \\ University of Mosul
}

Received

$08 / 12$ / 2011
Accepted

02 / 05 / 2012

\section{الخلاصة}

يؤثر تشتت النمط الاستقطابي دوراً كبيراً في أنظمة الاتصالات الضوئية عند معدلات

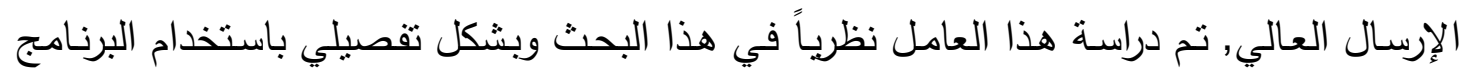

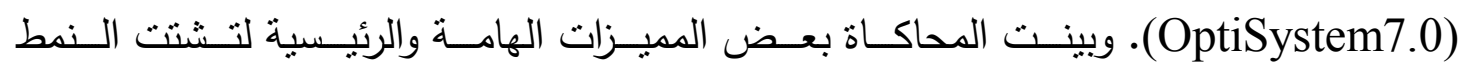

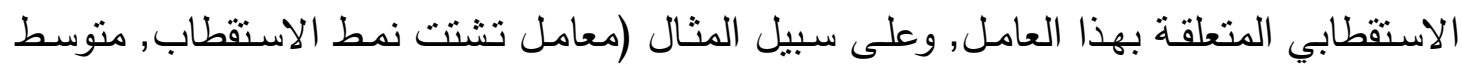

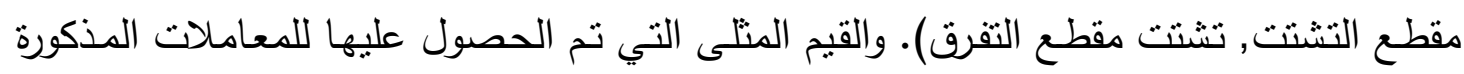

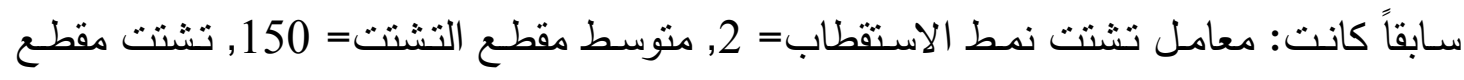
التفرق= 110.

تكمن أهمية هذه الدراسة لبيان تأثير العامل (PMD) عند معدل الإرسال العالي, والذي

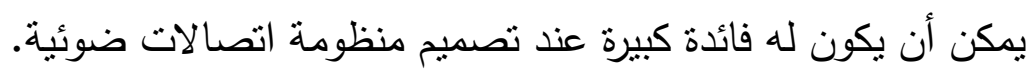

\begin{abstract}
Polarization Mode Dispersion (PMD) plays a great role in high bit rate optical communication systems. This factor had been studied thoroughly in this article of optical layout simulation by (OptiSystem7.0). This simulation had revealed some interesting features of (PMD) relating its major parameters, i.e, (PMD coefficient $\left(\mathrm{D}_{\mathrm{PMD}}\right)$ ), mean scattering section, and scattering section dispersion). An optimum values have found for the mentioned parameters were: $\mathrm{D}_{\mathrm{PMD}}=2$, mean scattering section $=150$, scattering section dispersion $=110$.

The opportunity to study the influence of PMD on high bit rate network can be of a substantial benefits to engineer the optical communication network.
\end{abstract}

\section{Introduction}

Polarization Mode Dispersion (PMD) is a major effect that has been encountered in the coherent optical communication and at high bit rate. 
This effect had been under high intensive efforts of scientists to solve this problem. In its simplest manifestation, (PMD) splits pulse between the fast and the slow axis in an optical fiber, at the same time. Higher orders of PMD induce depolarization and polarization-dependent chromatic dispersion (PCD) [1]. The (PMD) results from the variation in the refractive index and existence of the birefringence of the fiber with respect to the polarization of the light signal [2]. As result the polarization of the light in a waveguide travel at different speeds causing random spreading of optical pulses. This difficulty is ultimately limits the rate at which data can be transmitted over a fiber. In a realistic fiber, however, there are random imperfections that break the circular symmetry, causing the two polarizations to propagate with different speeds. In this case, the two orthogonal polarization components of a signal will slowly separate, e.g. causing pulses to spread and overlap. Because the imperfections are random, the pulse spreading effects correspond to a random walk [3]. The resulting difference in propagation times between the two polarization modes is known as differential group delay (DGD). Spinning the fiber during the drawing process reduces the differential group delay and hence the polarization mode dispersion [4], and thus have a mean polarizationdependent time-differential (also called the differential group delay or DGD) [3]. This fiber property is responsible for pulse broadening and, thus, a signal degradation, which can be assessed by the "effective pulse broadening" for single pulses or the "eye-opening penalty (EOP)"which is factor is defined as high quality factor (QF) and less bit error rate (BER), with regard to pulse sequences [5]. The bit error rate itself is insufficient for characterizing system performance. Adequate characterization requires introducing the probability distribution function (PDF) of the (BER) obtained by averaging over many realizations of birefringent disorder. Theoretical analysis showed that this PDF has an extended tail indicating the importance of anomalously large values of (BER) [6].

In this article, numerical investigated had been carried and for the effects of PMD on an optical communication layout, taking into account the fiber length, bit rate, and laser diode characteristics. (PMD) with its influence on the layout was studied in terms of (BER), quality factor (QF), gain, and signal power output.

\section{Theoretical Modeling}

Competitive market pressures demand that service providers continuously upgrade and maintain their network's to ensure they are able to deliver higher speed, higher quality applications and services to the customers. This requires verifying and ensuring that the network's fiber infrastructure and equipment can meet exacting performance standards and operate reliably [7].

Polarization Mode Dispersion (PMD) testing is becoming essential in the fiber characterization process, but still one of the most difficult 
parameter to test, due to its sensitivity to a number of environmental constraints [7].

The intensity of laser is Gaussian shape as function and given by [8]:

$\mathrm{I}(\mathrm{r}, \mathrm{z})=\mathrm{I}_{\mathrm{o}} \exp \left(-\frac{2 \mathrm{r}^{2}}{w_{o}^{2}}\right)$

Where: $(r)$ is the radial distance from the center axis of the beam, $\left(\mathrm{w}_{\mathrm{o}}\right)$ is the waist beam at $\left(\frac{\mathrm{I}}{\mathrm{I}_{\mathrm{o}}}=\frac{1}{\mathrm{e}^{2}}\right),(\mathrm{z})$ is the axial distance.

When a short pulse is launched through the fiber, a time delay with respect to the polarization mode delay will exist, and with its RMS width of the pulse $(\Delta \tau)$ given by [9]:

$(\Lambda \tau)^{2}=\frac{\xi^{2} h^{2}}{2}\left(\frac{2 \mathrm{z}}{\mathrm{h}}-1+\exp \left(-\frac{2 \mathrm{z}}{\mathrm{h}}\right)\right)$

is one half of the difference of the group delays per unit length between the fast and slow axes of the fiber, $(\xi)$ is a parameter that depends on its boundary condition, (h) is the mean coupling length.

In the limit $\mathrm{z}>>\mathrm{h}$ (high polarization mode coupling) with the laser pulse close to Gaussian, Equation (2) becomes [9]:

$(\Delta \tau)^{2}=\xi^{2} \mathrm{hz}$

The PMD coefficient parameter in the optical fiber $\left(\mathrm{D}_{\mathrm{PMD}}\right)$ component is given [9]:

$\left(\mathrm{D}_{\mathrm{PMD}}\right)=2 \xi \sqrt{\mathrm{h}}$.

where $\mathrm{D}_{\mathrm{PMD}}$ is the (PMD) parameter of the fiber, typically measured in $\mathrm{ps} / \sqrt{\mathrm{km}}$, a measure of the strength and frequency of the imperfections.

Given the (PMD) parameter, the average (DGD) of a fiber of length $\mathrm{L}$ is given by [2]:

$\mathrm{DGD}=\mathrm{D}_{\mathrm{PMD}} \sqrt{\mathrm{L}}$

the Bit Error Rate (BER) of transmitted data is given by [10]:

$\mathrm{BER}=\frac{1}{2} \operatorname{erfc}\left(\frac{\mathrm{QF}}{\sqrt{2}}\right)$

\section{Network layout}

To show the effect of PMD on characteristics of optical communication system, had been built this layout by using (OptiSystem7). The pulse generator consists of bit sequence source to generate binary signals $(0,1)$ and a Return to Zero (RZ) pulse generator. The laser diode was directly modulated and optical fiber to transmit the signal and photodetector (PIN) serving as a receiver which is connected with eye diagram analyzer to show the effects of (PMD) on the eye diagram and optical spectrum analyzer and dual port WDM analyzer. 


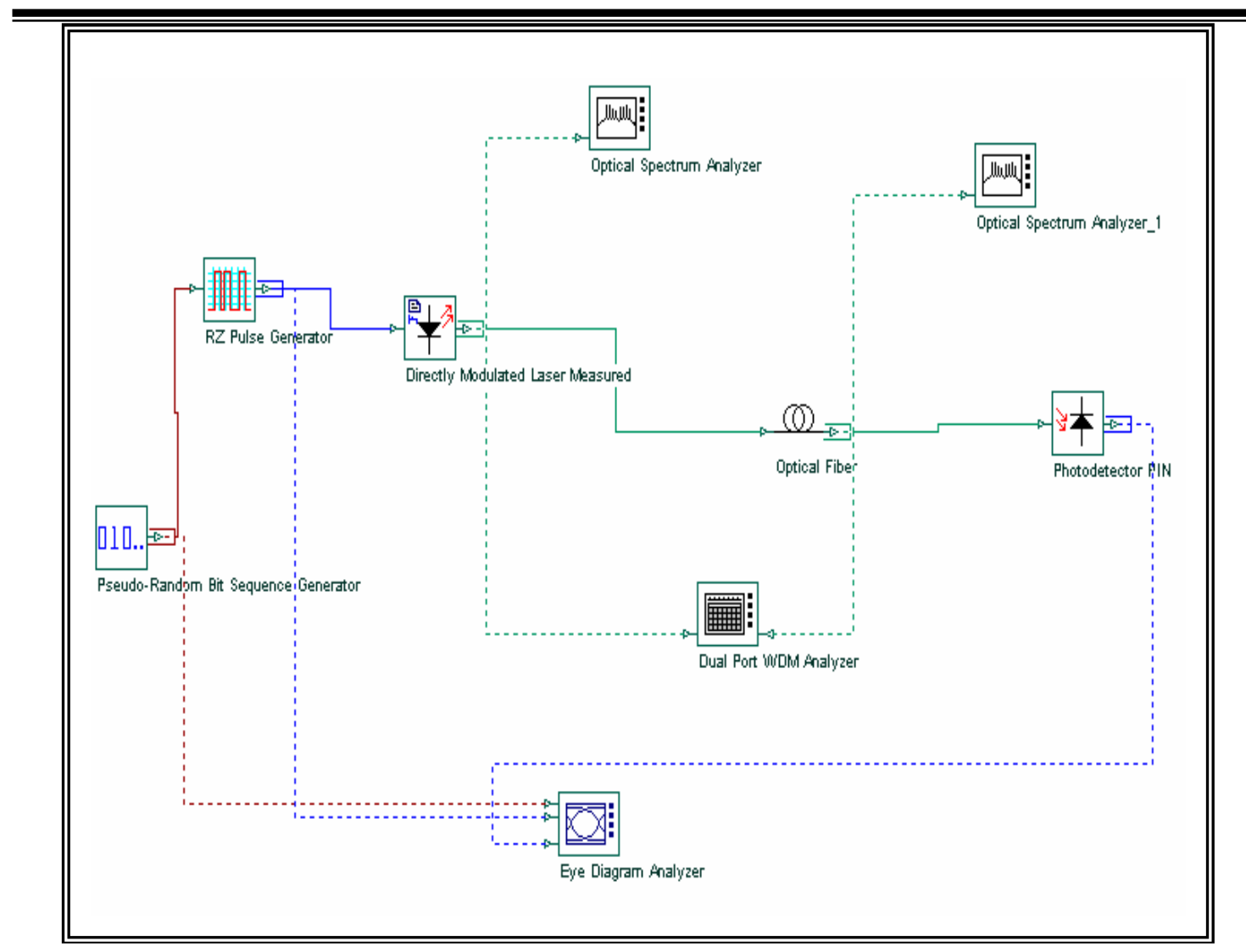

Figure (1): optical communication system to show effect of PMD

\section{Results and discussion}

The parameters that had been taken as optimum values in the optical communication system are show in Table (I) as:

Table (I): Main parameters of the optical communication system.

\begin{tabular}{|c||c||}
\hline \multicolumn{1}{|c||}{ Parameter } & Value \\
\hline \hline Laser wavelength & $1552.52 \mathrm{~nm}$ \\
\hline \hline Laser power & $5 \mathrm{~mW}$ \\
\hline \hline Length of fiber & $30 \mathrm{~km}$ \\
\hline \hline mean scattering section & $450 \mathrm{~m}$ \\
\hline \hline scattering section dispersion & $80 \mathrm{~m}$ \\
\hline \hline PMD coefficient & $10 \mathrm{ps} / \mathrm{sqrt}(\mathrm{km})$ \\
\hline \hline Bit Rate & $10 \mathrm{Gbit} / \mathrm{s}$ \\
\hline
\end{tabular}

As can be noticed from the table, the laser wavelength was adjusted in the minimum region of losses in the silica based fiber. The power of the laser diode was maintained at $5 \mathrm{~mW}$ when the spectrum was observed with low linewidth and single longitudinal mode. Fiber was taken as 30 $\mathrm{km}$ in order to operate without the need of optical amplifiers for these parameters. High bit rate of $10 \mathrm{Gbit} / \mathrm{s}$ for the transmission to be used in order to observe the effect of (PMD). 


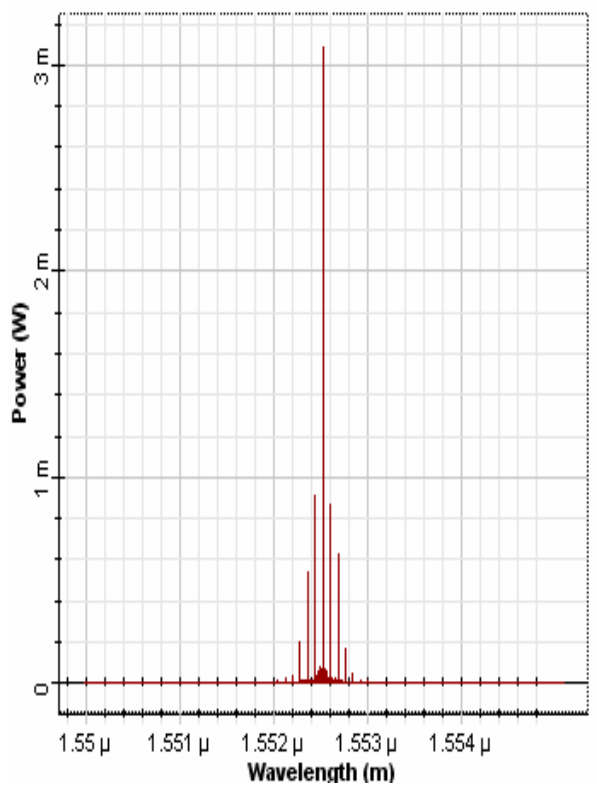

(a)

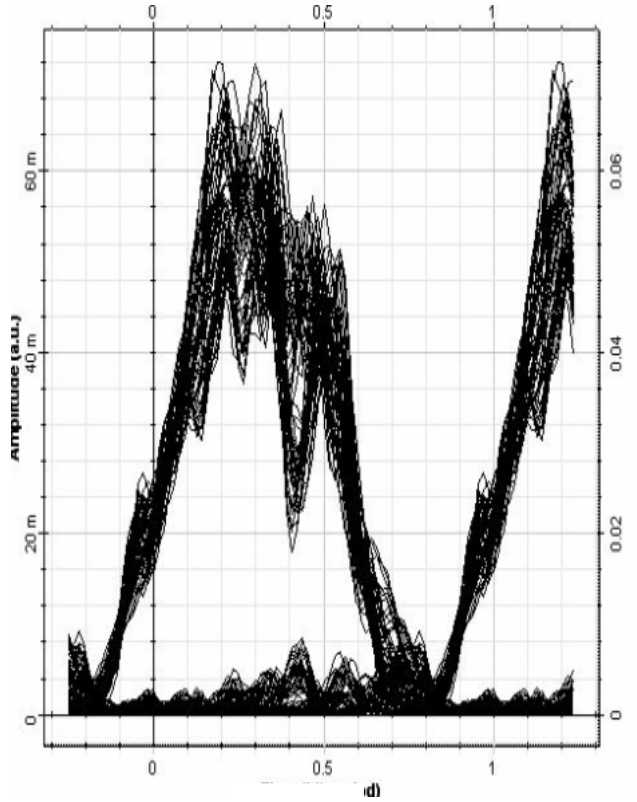

(h)

Figure (2): power output with PMD coefficient 10 ps/sqrt(km)

(a) optical spectrum

(b) eye Pattern

The quality of the eye diagram is related to severity of (PMD) effects, the eye opening and quality of the eye diagram decrease as (PMD) value and the bit-rate were increased. As it can be seen from the eye diagrams, with the same value of (PMD), but especially for low value of (DGD), it can be seen that the compensated signal perform an overcompensation that cause a distortion of the eye diagram[6].

We change the (PMD) coefficient to a value $7 \mathrm{ps} / \mathrm{sqrt}(\mathrm{km})$ and another value is shown in Table (I).

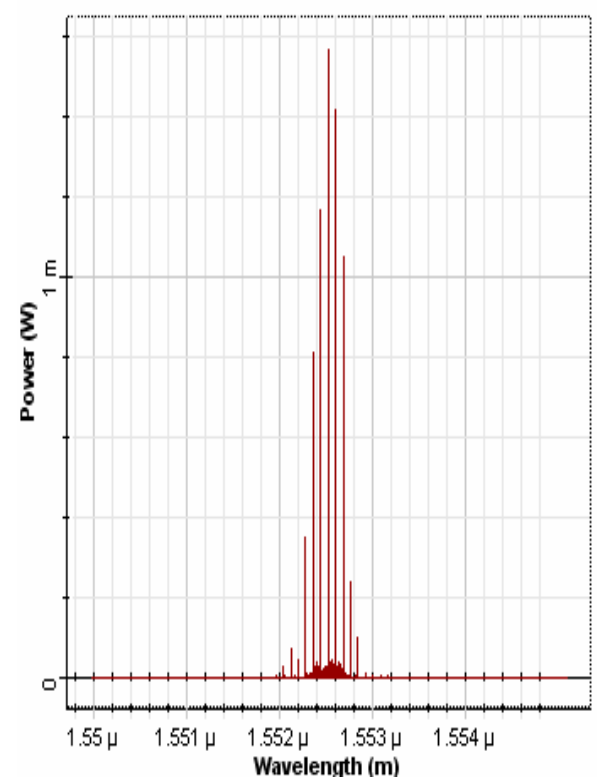

(a)

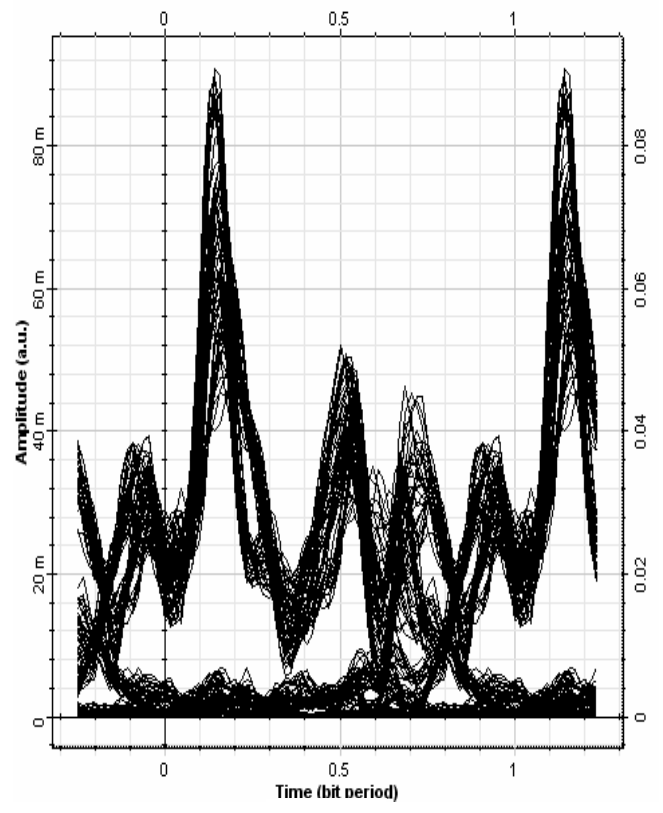

(b)

Figure (3): power output with PMD coefficient 7 ps/sqrt(km)

(a) optical spectrum

(b) eye Pattern 
we note that the increase of number of modes and will decrease the eye opining (decrease the QF and increase of BER) with decrease of PMD coefficient. as results in pulse broadening and distortion thereby leading to system performance degradation.

By changing the mean scattering section inside the optical communication system to value $(200 \mathrm{~m})$ and another value its shown in table (I), and the result is show in figure (4). many longitudinal modes had been observed due to the effects of increasing the scattering inside the optical fiber and due the effects of higher PMD

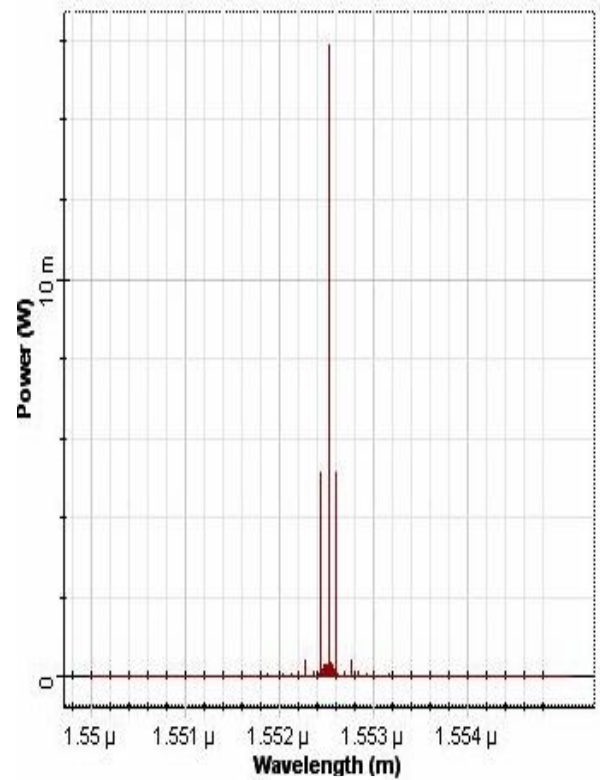

(a)

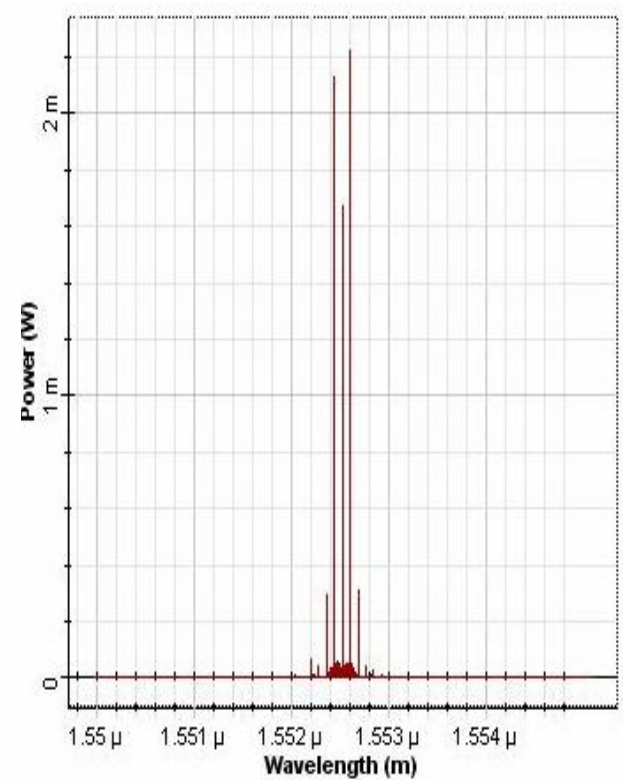

(b)

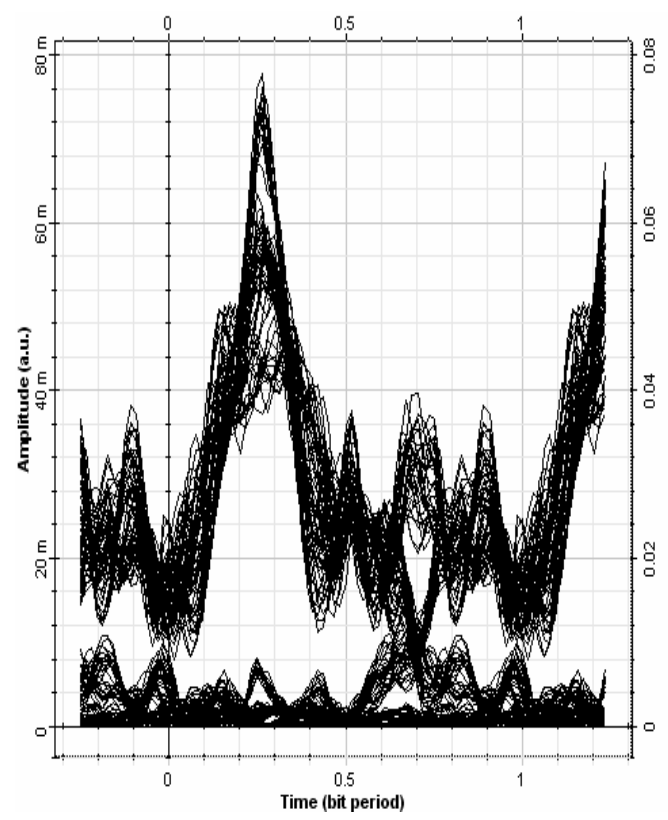

(c)

Figure (4): The effect of decreasing the mean scattering section $=200 \mathrm{~m}$ on
(a) transmitted optical spectrum
(b) received Optical spectrum
(c) eye Pattern 
The eye diagram of the received signal before compensation and after compensation shows a significant reduction in the degradation caused by PMD. Fig 4(a), and 4(b) show the eye diagram of the transmitted signal, received signal and compensated signal respectively [7].

In order to obtain an optimization values of (PMD) parameter such as: $\mathrm{D}_{\mathrm{PMD}}$, mean scattering section, and scattering section dispersion.

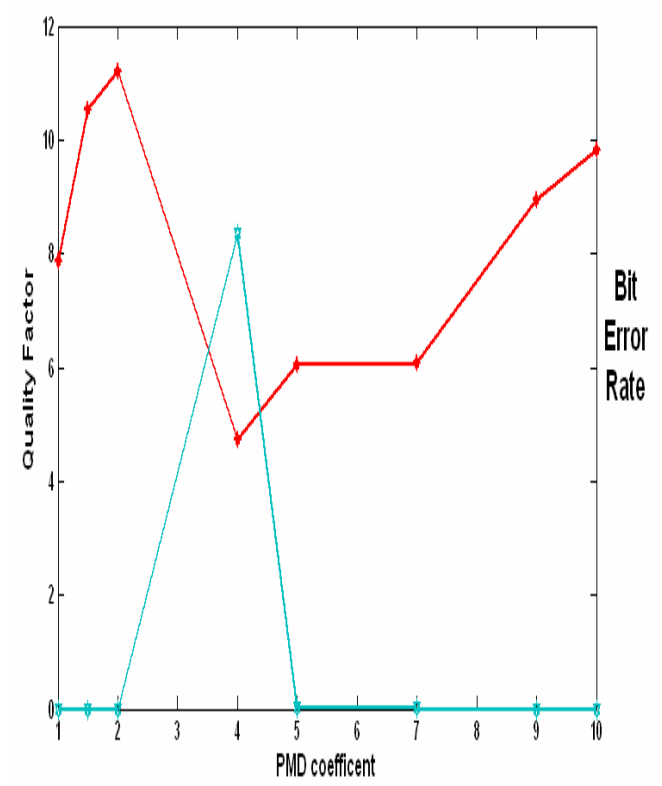

Figure (5): the relation between (QF, BER) and PMD coefficient

As can be noticed from the figure (6), (7), (8), we can say that (QF) is at optimum value when (BER) is in maximum value, this curtly the true of results according with equation (6).

And from the figure (6) we can say that to decrease the effect of (PMD) on optical communication system the best of the (PMD) coefficient is be $2 \mathrm{ps} / \mathrm{sqrt}(\mathrm{km})$ to given the best perform (optimum value) for its system.

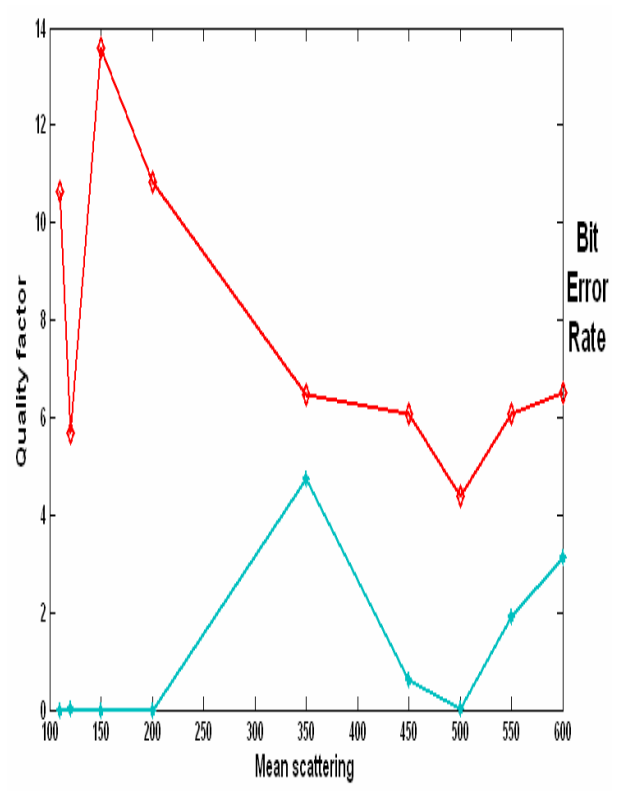

Figure (6): the relation between $(\mathrm{QF}, \mathrm{BER})$ and mean scattering section 
And from the figure (6) we can say that to decrease the effect of (PMD) on optical communication system the best of the mean scattering section is be $(150 \mathrm{~m})$ to given the best perform (optimum value) for its system.

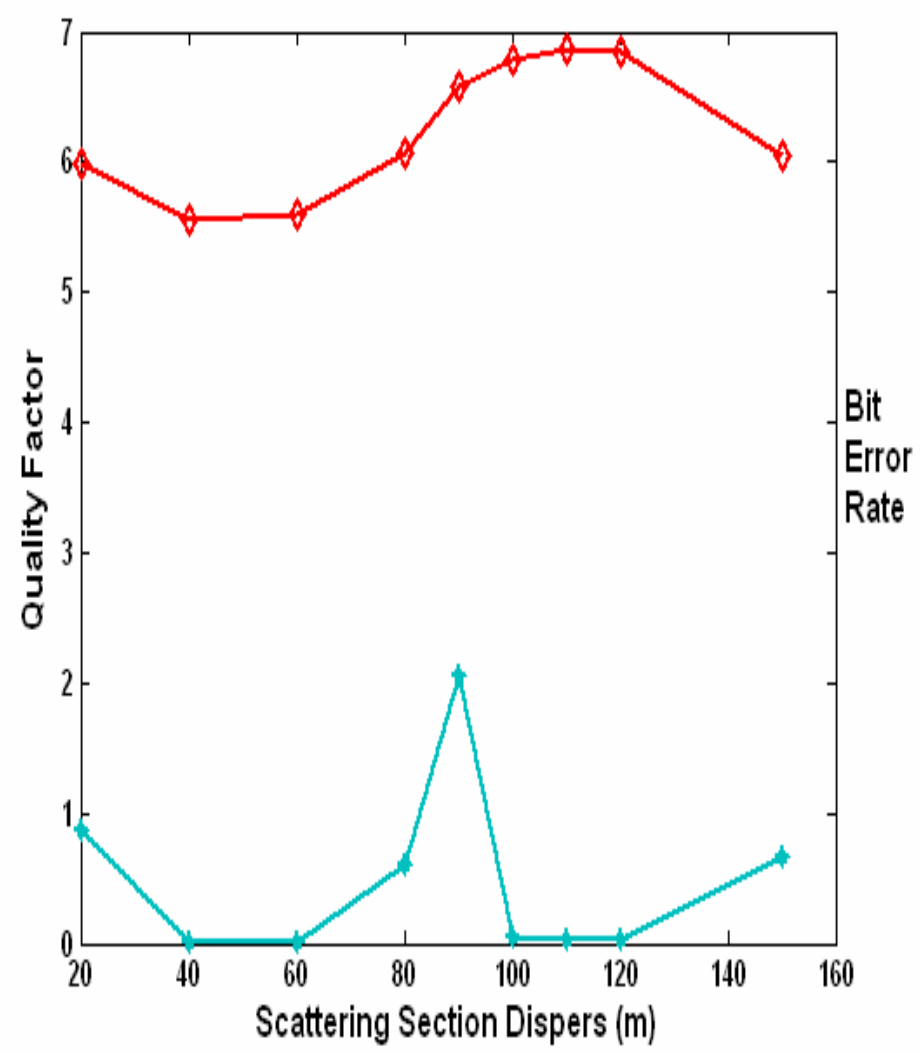

Figure (7): the relation between (QF, BER) and scattering section dispersion

And from the figure (7) in order to decrease the effect of (PMD) on optical communication system the best of the scattering section dispersion is be $(110 \mathrm{~m})$ to given the best perform(optimum value) for its system.

Table (II) The optimization values for PMD

\begin{tabular}{|c|c|c|}
\hline Parameter & Value & Unit \\
\hline \hline PMD coefficient & 2 & ps/sqrt(km) \\
\hline \hline mean scattering section & 150 & meter \\
\hline \hline scattering section dispersion & 110 & meter \\
\hline
\end{tabular}

The fitting equation of the plotted data is: $\Delta \tau=2 .(\xi)+2.7 \mathrm{e}-5$ with slope of (2). 


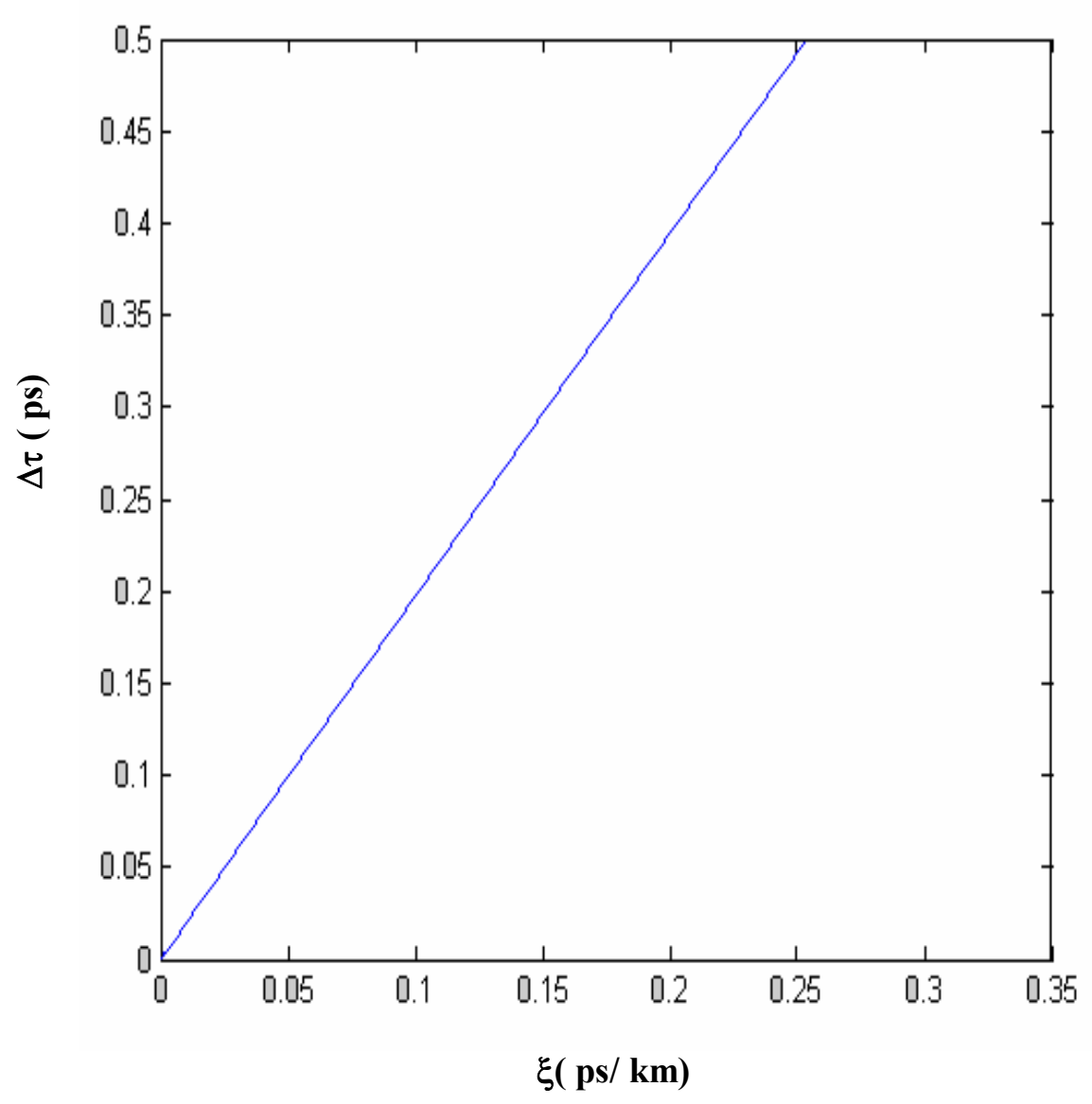

Figure (8): The relation between $(\Delta, \xi)$

According the condition $\mathrm{z}>>\mathrm{h}$, we plot $(\Delta \tau)$ versus $(\xi)$ by using equation (3) that will be true if $\left.\left.\left(\frac{\Delta \tau}{\Delta}\right\rangle\right\rangle 1\right)$ and the equation (3) should be linear as can be seen from figure ( 8$)$

\section{Conclusion}

Polarization mode dispersion (PMD) plays important role in high bit rate, coherent long haul optical fiber communication system. This study has revealed some important characteristics about the parameters of (PMD).

The results show that in order to minimize its influence, (PMD) parameters should be as given in Table (II). This table gives optimum values for (PMD).We can conclude that this theoretical study insight the major effects of PMD on quality factor(QF) and bit error rate (BER). 


\section{Reference}

1) Biondini, G., William, L., Member, K., And Curtis, R, (2004), "Importance sampling for polarization- mode dispersion: techniques and applications". J. Lightwave Techn. 22, 4.

2) Sewter, J., Carusone, A.C., Edward, S., "A comparison of equalizers for compensating polarization- mode dispersion in 40- Gb/s optical systems". Email: tcc@eecg.utoronto.ca.

3) Pilichi, A., (2010), "PMD impairments in optical fiber transmission at 10 Gbps and 40 Gbps". M.Sc. Thesis, Engineering faculty in Telecommunication Engineering.

4) Kumar, D. R., and Rao, P. B., (2010), "Polrization mode dispersion mitigation through spun fibers". Int. J. microwave. Opt. Techn .5,3.

5) Gravemann, T., Kissing, J., and Voges, E., (2004), "Signal degradation by second-order polarization-mode dispersion and noise". IEEE Photon Techn Letters. 16, 3.

6) Chernyak, V., Chertkov, M., Gabitov, I., Kolokolov, I., and Lebedev, V., (2004), "PMD-Induced fluctuations of bit-error rate in optical fiber Systems". J. microwave. Opt. Techn. 22, 4.

7) Lietaert, G., "Testing polarization Mode dispersion (PMD) in the field",www. fiberoptic. com.

8) Siegman, A.E., (1986), Lasers. University Science Books, Mill valley, California.

9) Gisin, N., Von der Weid, J. P., and Pellaux, J. P., (1991), "Optical fiber telecommunications IVB: systems and impairments". IEEE J.LightwaveTechn. 9, 7.

10) Agrawal, G. P., (2001), "Applications of nonlinear fiber optics", Academic Press, New York, 3rd edition. 